\section{Perfusion Imaging in Clinical Practice: A Multimodality Approach to Tissue Perfusion Analysis}

\section{F. Saremi}

New York, NY: Wolters Kluwer, 2015, 608 pages, $\$ 229.99$

With progressive and recursive iterations of tissue perfusion imaging and analytic techniques, it can be challenging to keep up with giant strides in advances in functional imaging and to apply practical implications of these diagnostic instruments to our daily clinical practice of examining disease states in various organs. What was historically secondarily inferred from large-vessel abnormalities and anatomic changes in organ-tissue architecture has been refined with the assessment of microvascular alterations reflecting changes earlier and with higher specificity. Tissue perfusion analysis has traditionally been the hallmark of myocardial and cerebral imaging. Newer image acquisition techniques and renewed exponential interest in applications have expanded this space of interest to include maxillofacial lesions; perfusion of the lung, prostate, kidneys, and liver; and cutting-edge applications in pediatrics, echocardiography, sonography, and musculoskeletal disease. This multiauthored text does not pay lip service to the newer echelons of perfusion imaging but has culled experiential educators to immediately jump into deep waters. Witness the classification of sonographic microbubble contrast agents based accordingly to the filling gas after barely a page of introduction: there is no need to separate the grain from the chaff here, but fret not: equations in physics and mathematic models cleverly appear exactly where the need for further disbursement of principles arises.

Whereas this book was no doubt chosen for review by this periodical because of the increasingly critical intersection of anatomic and molecular imaging, it is rewarding to note that the text is packaged highly systematically, with the first third of the chapters breaking down technical strategies such as dose reduction in myocardial perfusion imaging, reliability testing in CT and MR contrast-enhanced tissue perfusion imaging, MR perfusion assessment with susceptibility contrast, contrast enhancement, and arterial spin labeling. In addition to the unsurprising emphasis on the established body of peer-reviewed literature pertaining to myocardial and cerebral imaging, the inclusion of PET/SPECT and PET/MRI makes for a comprehensive collation of cutting-edge evidence-based guidelines. Sectional bibliographies are cosseted at the end of each of the 35 sections, again none of them hyperlinked to the source material-an opportunity that got away.

I strenuously recommend taking full advantage of the seamless multimedia edition with superior design of both content and navigation for both its inventiveness and its intuitive guidance. Having downloaded the content across both desktop and mobile platforms, I was able to seamlessly start with the hardcover and continue across devices from phone to tablet to desktop, making highlights and notes on another device, which can be cross-referenced retrospec-

COPYRIGHT (c) 2016 by the Society of Nuclear Medicine and Molecular Imaging, Inc. tively. Recognizing that a majority of our audience continues to favor print media, I did start and end with the hardcover book, which weighed just under 4 pounds and reinforced my bias for electronic media. Be aware that mouse clicks and scrolls are not correspondingly extrapolated to finger gestures, but that is not outside the industry standard. The search and share functions are robust, with highly detailed glossy full-color plates that are more anatomic than cartoon (to be fully appreciated, the tablet or desktop format was superior to the pinch and zoom on the smartphone). Regrettably, abbreviations both in the legends and in the image annotations are not immediately expanded but are clearly explained in the related textual narrative and highly abbreviated sliding panels - a conscious design choice that is not insuperable. For an example, see Figure 25-1: you would not know what it is meant to show if it migrated to another point in text or an entirely different chapter; that is to say, the illustrations and clinical examples do not stand on their own. A minor criticism is the low-pixel-density resolution of awkwardly typeset tables that quickly improves when boosted to full-screen magnification. Attempts to return to the placeholder on a magnified image loads the top of the section or subsection instead of the image per se, a design flaw I hope will be addressed in future editions. Clicking on superscripted citation numbers does not hyperlink to the source article or its abstract but merely displays the title of the citation. Sections within chapters are of dissimilar length and content density, a natural by-product of more than 80 contributing authors but barely distracting when assessing material electronically. The introduction to arterial spin labeling (Chapter 14) is a coy 5 lines long, making this quite the page turner with all the urgency of a Professor Robert Langdon adventure. This barely registers in the e-book edition because novices will certainly relish tossing back and forth between principles, artifacts, and clinical illustrative examples rather than juggernauting straight through as your reviewer did. For an online-based resource, there was a paucity of interactive work, be it in the form of learningbased quizzing, multimodality fusion overlays, or cinematic displays; the latter would enhance the experience of asynchronous learning as a mere substitute that mirrors everyday clinical work.

Perfusion Imaging in Clinical Practice is recommended as a palate cleanser for all practitioners of diagnostic imaging who find themselves at the crossroads of structure and function so that together we may get at the answers we seek with good evidence, rapidity, and outcomes. Be advised that in a driving range with so much activity and forward motion, there can be no one expansive, authoritative ex cathedra font of facts though there will always be a work such as this which quenches you but not to the point of satiety. Once stirred to know more by Dr. Saremi et al., we will be sure to seek it out.

\author{
Sundeep M. Nayak \\ Kaiser San Leandro Medical Centre \\ 2500 Merced St. \\ San Leandro, CA 94577 \\ E-mail: sundeep.m.nayak@kp.org
}

Published online Dec. 10, 2015.

DOI: 10.2967/jnumed.115.171025 